\title{
Comprehensive Time-Course
} Transcriptome and Co-expression Network Analyses Identify Salt Stress Responding Mechanisms in Chlamydomonas reinhardtii Strain GY-D55

\section{OPEN ACCESS}

Edited by:

Vinay Kumar,

Pune University, India

Reviewed by:

Jelli Venkatesh,

Seoul National University,

South Korea

Mohammad Israil Ansari,

University of Lucknow, India

Akhilesh Kumar Pandey,

Banaras Hindu University, India

*Correspondence:

Xue-Jie Zhang

zhangxuejie@sdnu.edu.cn Shou-Jin Fan

fansj@sdnu.edu.cn

Specialty section:

This article was submitted to

Plant Abiotic Stress,

a section of the journal

Frontiers in Plant Science

Received: 03 December 2021

Accepted: 10 January 2022

Published: 24 February 2022

Citation:

Zhang $L-Y$, Xing Z-T, Chen $L-Q$,

Zhang X-J and Fan S-J (2022)

Comprehensive Time-Course

Transcriptome and Co-expression Network Analyses Identify Salt Stress

Responding Mechanisms

in Chlamydomonas reinhardtii Strain

GY-D55. Front. Plant Sci. 13:828321.

doi: 10.3389/fpls.2022.828321

\section{Luo-Yan Zhang, Zhao-Tian Xing, Li-Qian Chen, Xue-Jie Zhang* and Shou-Jin Fan*}

Shandong Provincial Key Laboratory of Plant Stress Research, College of Life Sciences, Shandong Normal University, Jinan, China

It is highly necessary to understand the molecular mechanism underlying the salt stress response in green algae, which may contribute to finding the evolutionary cues of abiotic stress response in plants. Here, we reported a comprehensive temporal investigation of transcriptomes using data at eight different time points, from an early stage ( $2 \mathrm{~h}$ ) to a late stage (up to 96 h) in Chlamydomonas reinhardtii GY-D55 cells. The principal component analysis (PCA) of transcriptome profiles showed that the samples of the early and late stages were well separated. A total of 12,445 genes were detected as differentially expressed genes. There were 1,861/2,270 common upregulated/downregulated genes for each time point compared with control samples. Samples treated with salt for 2 , 8 , and $24 \mathrm{~h}$ had a relatively large number of characteristic upregulated/downregulated genes. The functional enrichment analysis highlighted the timing of candidate regulatory mechanisms for salt stress responses in GY-D55 cells. Short time exposure to salt stress impaired oxidation-reduction, protein synthesis and modification, and photosynthesis. The algal cells promoted transcriptional regulation and protein folding to deal with protein synthesis/modification impairments and rapidly accumulated glycerol in the early stage $(2-4 \mathrm{~h})$ to cope with osmotic stress. At 12 and 24 h, GY-D55 cells showed increased expressions of signaling and photosynthetic genes to deal with the damage of photosynthesis. The co-expression module blue was predicted to regulate endoplasmic reticulum (ER) stress at early time points. In addition, we identified a total of 113 transcription factors (TFs) and predicted the potential roles of Alfin, C2C2, and the MYB family TFs in algal salt stress response.

Keywords: Chlamydomonas reinhardtii, time-course transcriptome, salt stress, co-expression network, strain GY-D55

\section{INTRODUCTION}

As one of the most critical abiotic stresses, high salinity greatly impairs agricultural productivity globally. Although plants have progressively acquired a variety of adaptive molecular, physiological, and biochemical mechanisms to deal with salinity stress, it can still affect $30 \%$ of arable soils by 2050 (Munns and Tester, 2008). The physiological/biochemical regulation, transcription/translation 
machinery, and epigenetic changes may trigger ecological adaptation (such as salt stress adaptation), while these changes over time can remarkably affect species phenotypes and distributions (Yang et al., 2019; Funck et al., 2020; Verdu et al., 2021). Gene-rich mixotrophic lineages, such as Chlamydomonas, can quickly adapt to either climate change-induced or physical abiotic stress. Therefore, it is necessary to explore the molecular mechanism underlying the salt stress response in green algae, which may contribute to identifying the evolutionary cues of abiotic stress response in plants and developing salt-resistant crops with valuable salt-responding characteristics (Husic and Quigley, 1990; Perrineau et al., 2014; Gierz et al., 2017; Wang et al., 2018, 2020; Yang et al., 2020).

Different organisms adapt to extreme surrounding conditions via various mechanisms, and understanding these mechanisms can help explore the key features of biological processes (BPs), including the biochemical limits to macromolecular stability and the genetic instructions for constructing macromolecules that stabilize under one or more extreme conditions (Grant et al., 1998; Luo and Liu, 2011; Foflonker et al., 2014). Generally speaking, there are two basic mechanisms in halophiles: (i) "salt-in" strategy: halophilic algae accumulate $\mathrm{KCl}$ in their cells to keep high intracellular salt levels, osmotically at least equivalent to the external levels; (ii) other halophilic algae create or accumulate osmoregulation substances that have osmotic potential. The majority of green algae (Chlorophyceae) are moderately halophilic. However, a few algae, including Dunaliella salina, are extremely halophilic species (Grant et al., 1998; Gammoudi et al., 2021). These species are responsible for most of the primary production when exposed to high salt stress (Oren, 2005). Nevertheless, most freshwater strains show retarded development and impaired survival when exposed to high salinity, and these strains are generally unable to survive beyond a low threshold of salinity. Various pathways are used by these salt-sensitive algae to deal with salt stress. Glycolysis, the Kennedy pathway, and the Calvin cycle are all adopted to enhance both osmolyte production and reserves of storage molecules, mostly lipids (Shetty et al., 2019). A previous study on saltadapted mutants of Chlamydomonas reinhardtii has shown that algae can adapt to loss in photosynthetic efficiency by taking up acetate to enhance energy generation and carbon assimilation (Perrineau et al., 2014).

As a free-living freshwater green alga, the unicellular alga C. reinhardtii is a well-characterized model alga, and its full genome has been sequenced. This alga is widely used to investigate stress-inducible responses since it carries a series of stress-related genes. However, the functional significance of these genes in adaptive metabolism remains largely undetermined (Mendez-Alvarez et al., 1999; Grossman, 2000; Perrineau et al., 2014). C. reinhardtii has been used to assess responses to a series of abiotic stress agents, including salinity, temperature, irradiation, nutrient starvation, and heavy metals (Hanikenne, 2003; Pollock et al., 2005; Mahong et al., 2012; James et al., 2013; Neelam and Subramanyam, 2013; Perrineau et al., 2014; Erickson et al., 2015; Breker et al., 2016).

Existing research on $C$. reinhardtii shows that at the morphological level, salt stress of 150 or $200 \mathrm{mM} \mathrm{NaCl}$ results in flagellar resorption, decreased cell size, slower growth rate, and palmelloid morphology (Neelam and Subramanyam, 2013; Khona et al., 2016). Salt stress metabolically affects the demand for energy to keep ion homeostasis, the scavenging of reactive oxygen species (ROS) by antioxidant enzymes, and the upregulation of heat shock proteins (HSPs) and chaperones to aid protein renaturation due to misfolding or aggregation (Yokthongwattana et al., 2012; Sithtisarn et al., 2017). Salinity composes stress by damaging ionic and osmotic balances in plants. Algal cells with inflexible cell walls have limited ability to change cell volume and thus depend heavily on organic solutes for osmoregulation. Glycerol, proline, and trehalose with a neutral charge and low toxicity at high concentrations are usually used as compatible solutes. Glycerol is an effective compatible solute that is produced by most salt-sensitive algal species under high saline stress. In Chlorella autotrophica (Ahmad and Hellebust, 1984), C. reinhardtii (Perrineau et al., 2014), Chlamydomonas mexicana (Salama el et al., 2013), Chlamydomonas pulsatilla, and Chlamydomonas sp. JSC4 (Ho et al., 2017) showed an increase in glycerol content in response to increasing salinity. Besides, salt stress usually induces the expression of many transcription factors (TFs), which then activate the expression of numerous downstream osmotic and ionic stress responding genes (Gong et al., 2020). In C. reinhardtii, basic leucine region zipper (bZIP) TFs play important roles in mediating photosynthesis and lipid accumulation of alga in response to stresses. Several members from bZIP, C3H, Dof, and MYB TFs families were differentially expressed throughout the entire time course in cold stress responding (Li et al., 2020).

Some studies have reported that different strains of this green alga have various salt stress responses. In C. reinhardtii, a retarded cell growth and weaker photosynthesis are observed after a short time of exposure to sodium chloride $(\mathrm{NaCl})$, whereas the growth and photosynthetic activity in several strains of $C$. reinhardtii recover over $24 \mathrm{~h}$ (Perrineau et al., 2014). In a long-term selection study, evolved C. reinhardtii populations can grow rapidly in a high salt medium $(200 \mathrm{mM} \mathrm{NaCl})$ as progenitor cells (Perrineau et al., 2014). These C. reinhardtii cells display well-known responses to salt stress, such as reduced photosynthesis, enhanced glycerophospholipid signaling, and activated transcription and translation machinery. Quantitative proteomic comparison of salt stress in C. reinhardtii strain CC-4325 and the snow alga Chlamydomonas nivalis reveals that salt stress enhances the accumulation of fatty acids in the un-sequenced alga $C$. nivalis as it switches to a non-growth state, while $C$. reinhardtii does not have this response (Hounslow et al., 2021). In our previous study, we have predicted the molecular and genetic mechanisms of salt stress responses in C. reinhardtii strain GY-D55 after short-term acclimation to salt stress (200 $\mathrm{mM} \mathrm{NaCl}$ for $24 \mathrm{~h}$ ) (Wang et al., 2018).

It is highly possible that many genes that are important for the salt stress responding in the $C$. reinhardtii strain GY-D55 (but are expressed only in a short period of time) have not been identified yet, due to the fact that the previous proteomic and transcriptomic studies did not have sufficient temporal resolution. Here we report a comprehensive temporal 
investigation of transcriptomes using data for eight time points, from early stage ( $2 \mathrm{~h}$ ) to late stage (up to $96 \mathrm{~h}$ ). This time-course transcriptome analysis aims to highlight the timings of candidate regulatory genes and mechanisms for salt stress responses in GYD55 cells.

\section{MATERIALS AND METHODS}

\section{Algal Material Preparation, Stress Treatments, and Morphological Observations}

The wild-type C. reinhardtii strain GY-D55 was obtained from LeadingTec Co., Ltd. ${ }^{1}$ and maintained in $150 \mathrm{ml}$ of BG-11 medium on a shaking table at $120 \mathrm{rpm}$ under the following conditions: photoperiod 16-8 h/light-dark, temperature $23^{\circ} \mathrm{C}$, and light intensity $100 \mu \mathrm{mol} \mathrm{m} \mathrm{m}^{-2} \mathrm{~s}-1$. Under the abovementioned conditions, $C$. reinhardtii cells were cultured in a BG11 medium for approximately 14 days until the cell density reached $2 \times 10^{6}$ cells $/ \mathrm{ml}$. The cells of the mid-logarithmic phase were used for salt stress treatment referring to the study published by Khona et al. (2016).

It was necessary to point out how we set up the time points of salt stress in this study. The existing studies have suggested that there are certain dynamic changes in the phenotype, physiology, and biochemistry affected in C. reinhardtii cells by salt stress at different time points, which helped us design time points. In the study published by Vega et al. (2006), the photosynthetic activity in C. reinhardtii is initially $(1-2 \mathrm{~h})$ blocked by the addition of $200 \mathrm{mM}$ salt. During $4-12 \mathrm{~h}$, the decreasing trend of photosynthetic activities slowed down and respiratory activities began to recover gradually (Vega et al., 2006). These findings suggest that there is a dynamic inhibitory effect on the photosynthesis in C. reinhardtii during 2-12 h. A duration of $24 \mathrm{~h}$ is the key time point for the alga to respond to salt stress, and the photosynthetic activity of several strains recovers over $24 \mathrm{~h}$ (Vega et al., 2006; Crespo, 2012; Erickson et al., 2015). In the study published by Ji et al. (2018), the total lipid content in $C$. reinhardtii cells is significantly increased when exposed to salt stress $(150 \mathrm{mM})$ for $48 \mathrm{~h}$. Besides, $200 \mathrm{mM} \mathrm{NaCl}$ treatment inhibits the growth and significantly increases the lipid content of $C$. reinhardtii at 3 days (72 h) (Hang et al., 2020). Salt treatment strikingly induces the production of nitric oxide (NO) after 1-3 days (24-72 h), suggesting that $\mathrm{NO}$, as another second messenger, triggers salt stress response (Chen et al., 2016; Bazzani et al., 2021). Besides, the salt stress gradually induced the activities of antioxidant enzymes after 1 or 3 days $(72 \mathrm{~h}$ ) of salt treatment, and such induced activities were declined after 3 days (72-96 h) of salt treatment. Therefore, it was reasonable to set an eight-time-point regimen $(2,4,8,12,24,48,72$, and $96 \mathrm{~h})$ to explore the molecular mechanisms of photosynthesis impairments and the suppression of ROS and lipid metabolism in salt stress response in $C$. reinhardtii cells.

\footnotetext{
${ }^{1}$ http://www.leadingtec.cn/
}

The $\mathrm{NaCl}$ treatment was carried out as previously described by Wang et al. (2018). Briefly, a $50 \mathrm{ml}$ medium containing $800 \mathrm{mM} \mathrm{NaCl}$ was added to a $150 \mathrm{ml}$ culture medium, the final $\mathrm{NaCl}$ concentration was $200 \mathrm{mM}$, and the $\mathrm{pH}$ of the medium was adjusted to 7.0. Cells cultured in the absence of $\mathrm{NaCl}$ were used as the control group. Each experiment was conducted in triplicate. The cells of $C$. reinhardtii were collected at eight preset time points after exposure to $200 \mathrm{mM} \mathrm{NaCl}: 2,4,8,12,24,48$, 72 , and $96 \mathrm{~h}$. To evaluate the morphological changes, including the difference in cell size, salt stress-induced palmelloids, or chlorophyll bleaching under stressful conditions, a $50 \mu \mathrm{L}$ sample was observed using an Olympus SZ61 microscope (Olympus Instruments Inc., Tokyo, Japan) at $40 / 100 \times$ magnification. The $t$-test analysis was used to evaluate the growth ratio between cells under control and saline condition. The cell count of algal material was estimated at eight time-points and relative growth rates $(\mathrm{RGR})$ were calculated: $\mathrm{RGR}=(\ln \mathrm{N} 2-\ln \mathrm{N} 1) / \Delta \mathrm{t} \times 100 \%$, where N1 is the cell count at time-point 1 and N2 is the cell count at time-point $2, \Delta \mathrm{t}$ is the time interval (hours). The statistical differences between salt stress and control samples were analyzed by the one-way ANOVA in R.

\section{RNA Extraction, Illumina Library Construction, and Sequencing}

Briefly, $100 \mathrm{ml}$ cell cultures from different groups were subjected to centrifugation at $3,000 \mathrm{~g}$ for $5 \mathrm{~min}$, and the cell pellet was resuspended in $25 \mathrm{ml}$ RNAlater solution (Ambion). The total RNA was isolated using the TRIzol Reagent (Invitrogen) according to the manufacturer's instructions. The integrity of RNA was evaluated using the RNA Nano 6000 Assay Kit of the Agilent Bioanalyzer 2100 system (Agilent Technologies, CA, United States) and the NanoDrop 2000 spectrophotometer (Thermo Scientific, Wilmington, MA, United States).

Sequencing libraries were constructed using the NEBNext ${ }^{\circledR}$ UltraTM RNA Library Prep Kit for Illumina ${ }^{\circledR}$ (NEB, United States) according to the manufacturer's instructions, and the sequences were attributed to each sample based on the index codes. Briefly, total RNA was subjected to purification using poly-T oligo attached magnetic beads. Purified messenger RNA (mRNA) was reversely transcribed into first-strand complementary DNA (cDNA) using a random hexamer primer and $\mathrm{M}$ MuLV Reverse Transcriptase $\left(\mathrm{RNase}^{-}\right)$. DNA Polymerase I and RNase $\mathrm{H}$ were adopted for the synthesis of the second-strand cDNA. cDNA fragments of 370-420 bp were subjected to purification using the AMPure XP system (Beckman Coulter, Beverly, MA, United States). The size selected, adaptor-ligated fragments were purified isolated and enriched by PCR. The resulting products were subjected to high throughput sequencing using an Illumina HiSeq $\mathrm{X}$ platform (Illumina, San Diego, CA, United States). All genetic data have been submitted to the NCBI Sequence Read Archive (SRA) database, ${ }^{2}$ PRJNA770825 and the Gene Expression Omnibus (GEO) database, ${ }^{3}$ GSE191218.

\footnotetext{
${ }^{2}$ https://submit.ncbi.nlm.nih.gov/subs/sra

${ }^{3}$ https://www.ncbi.nlm.nih.gov/geo/
} 


\section{RNA Sequencing and Read Mapping of the Chlamydomonas reinhardtii Transcriptome}

The RNA sample of every accession was sequenced by the Illumina NovaSeq 6000. The cDNA library was constructed, and Illumina pair-end 150 bp sequencing (PE150) was carried out at Novogene Co., $\mathrm{Ltd}^{4}$ Raw reads of the fastq format were firstly processed through in-house perl scripts. In this step, the adapter containing reads, ploy- $\mathrm{N}$ containing reads, and low-quality reads were removed from raw data, yielding clean reads. Meanwhile, the Q20, Q30, GC content, and sequence duplication levels of the clean data were determined. All the downstream analyses were conducted using high-quality clean data. Reference genome ${ }^{5}$ and gene model annotation files ${ }^{6}$ were downloaded from the genome website directly. The index of the reference genome was built using Hisat2 (v2.0.5), which was adopted to align pairedend clean reads to the reference genome. For the prediction of novel transcripts, StringTie (v1.3.3b) was adopted to assemble the mapped reads of each sample as previously described (Pertea et al., 2015).

\section{Calculation of Gene Expression in Chlamydomonas reinhardtii}

In the present study, 27 independent cDNA libraries were constructed for individual samples of $C$. reinhardtii using a PE150 sequencing analysis. The number of reads mapped to each gene was determined using the featureCounts v1.5.0 p3. Subsequently, the length of the gene and read count mapped to this gene were used to calculate the fragment per kilobase of exon model per million mapped reads (FPKM) of each gene. Differential expression analysis of salt-treated and control samples at different time points was performed using the DESeq2 R package (Love et al., 2014). The false discovery rate was controlled by adjusting the $P$-value by Benjamini and Hochberg's approach. Differentially expressed genes (DEGs) were defined using thresholds of $P$-value $<0.05$ and $\mid \log 2$ (foldchange) $\mid>0$ and 1. The union of the DEGs was analyzed by the heatmap package of $\mathrm{R}$ for gene cluster construction, using the Euclidean distance clustering algorithm. The normalized expression values of DEGs were calculated by dividing their expression level at different time points with their maximum observed FPKM.

\section{Weighted Gene Co-expression Network Analysis Co-expression Network Construction}

The FPKM values of genes were transformed and normalized to construct the weighted gene co-expression network analysis (WGCNA) co-expression network. The genes with a low variance of expression values among samples were omitted.

\footnotetext{
${ }^{4} \mathrm{http}: / /$ www.novogene.com/

${ }^{5} \mathrm{ftp} / / / \mathrm{ftp}$. ensemblgenomes.org/pub/release-39/plants/fasta/chlamydomonas_ reinhardtii/dna/

${ }^{6} \mathrm{ftp} / / / \mathrm{ftp}$.ensemblgenomes.org/pub/release-39/plants/gtf/chlamydomonas_ reinhardtii/
}

A co-expression network for the filtered genes was constructed using the WGCNA package in R (Version 3.3.2) (Zhang and Horvath, 2005). After sample clustering, the soft threshold of module analysis was determined using the scale independence and mean connectivity analysis of modules with different power values. The power value ranged from 1 to 20 , and then the values of scale independence and mean connectivity were calculated accordingly. The power value was calculated when the scale independence value was 0.9 . To classify the similar gene expression profiles into different gene modules, the average distance with a minimum size threshold of 30 and the merge cut height of 0.25 were used to construct a hierarchical clustering dendrogram of the TOM matrix. Moreover, a cluster dendrogram among modules and an eigengene adjacency heatmap between modules were generated. Cytoscope 2.8.2 Shannon et al. (2003) was used to visualize the co-expression networks. Significant co-expression modules related to the salt stress response (as a trait) were identified using the information time-course $\mathrm{NaCl}$ treatment of 27 samples. The correlation between modules and traits was used to calculate the module-trait relationships, and the modules significantly associated with individual traits $(P$-value $<0.05)$ were identified.

\section{Gene Ontology Enrichment and Transcription Factor Screening}

Based on the sequence similarity against the genome of Arabidopsis thaliana with an $E$-value cut-off of $1 \mathrm{e}^{-5}$, the genes of C. reinhardtii were mapped to $A$. thaliana gene IDs accordingly. The TF data of $A$. thaliana were downloaded from plantTFDB. The homologous of $A$. thaliana TFs in C. reinhardtii were screened as TFs in C. reinhardtii strain GY-D55. The Gene Ontology (GO) enrichment analysis for DEGs of different clusters and genes of co-expression modules was conducted by the topGO package of $\mathrm{R}$.

\section{Quantitative Reverse Transcription PCR for Data Validation}

Eight different genes were selected to verify the RNA-Seq result, including four upregulated genes (Pyrroline-5carboxylate synthetase coding gene CHLREDRAFT_130812, Gal oxidase coding gene CHLREDRAFT_196816, Myb family TF CHLREDRAFT_205725, and WRKY family TF CHLREDRAFT_205718) and four downregulated genes (UDPsulfoquinovose synthase coding gene CHLREDRAFT_27658, oxygen-evolving enhancer protein 2 of photosystem II coding gene CHLREDRAFT_33411, catalase coding gene CHLREDRAFT_190083, and L-ascorbate peroxidase coding gene CHLREDRAFT_192806). Gene sequences were extracted, and their primers were designed using Primer 5 software (Lalitha, 2000). RNA extraction and quality tests were performed as described above, and quantitative reverse transcription PCR (qRT-PCR) was performed according to the method described by Wang et al. (2018). Using the homolog of the

\footnotetext{
${ }^{7}$ http://planttfdb.cbi.pku.edu.cn/
} 
GTP binding elongation factor Tu family protein EF1ALPHA (CHLREDRAFT_132905) as a reference internal gene. qRT-PCR was performed using SYBR Green qRT-PCR Master Mix (DBI, Ludwigshafen, Germany) in ABI7500 Real-Time PCR System (ABI, Waltham, MA, United States). Three replicates were performed, and the amplicons were used for melting curve analysis to evaluate the amplification specificity. Relative gene expression was quantified using the $2^{-\Delta \Delta \mathrm{Ct}}$ method. The statistical differences between samples were analyzed by one-way ANOVA in $\mathrm{R}$. The correlation coefficient of gene expression between qRT-PCR analysis and RNA-Seq data was analyzed in R.

\section{RESULTS}

\section{Effect of Salt on Chlamydomonas reinhardtii Strain GY-D55 Cell Growth}

Growth analyses based on cell density showed that the growth rate of $C$. reinhardtii strain GY-D55 was detrimentally affected in the presence of $200 \mathrm{mM} \mathrm{NaCl}$ (Figure 1A and Supplementary Table 1). Moreover, the $44 \%$ reduction of growth rate was observed under the salt conditions at 0-96 h. Figure 1B shows that the RGR of the $\mathrm{NaCl}$-treated and control groups in $0-2 \mathrm{~h}$ was significantly different $(P<0.01)$. In the NaCl-treated group, the RGR was negative during this period, indicating that the growth of GY-D55 cells was significantly inhibited in the early stage of salt stress. During 8-12 h, although the RGR of algae under salt stress was still significantly lower $(P<0.05)$ compared with the control group, GY-D55 began to gradually increase the number of cells during this period. After $48 \mathrm{~h}$, the RGR of GY-D55 cells under salt stress was higher compared with the control group, although both values were very small. Besides, $200 \mathrm{mM} \mathrm{NaCl}$ exposure GY-D55 cells showed the time-dependent formation of palmelloids (Figures 1C-F). Two cell clusters were observed in the $4-8 \mathrm{~h}$ salt-treated samples (Figure 1D), four-celled clusters (akin to palmelloids) were found in cells under the $24-48 \mathrm{~h} \mathrm{NaCl}$ treatment (Figures 1E,F), chlorophyll bleaching was found at the 2-12 h salt stress treated cells.

\section{Transcriptome Profiling and Novel Transcripts Discovery}

The total mRNA from the cells of the three control and 24 salt stress treated samples of C. reinhardtii strain GY-D55 were sequenced using the Illumina system. The pairend reads obtained from 27 samples of C. reinhardtii were shown in Supplementary Table 2. In total, we obtained more than 100 million raw reads for each C. reinhardtii sample with at least 60 million reads for each condition and time point. More than $89 \%$ of the HQ reads from the individual sample (control and salinity) of $C$. reinhardtii strain GY-D55 could be mapped on the C. reinhardtii genome (Supplementary Table 2). The assembly of mapped reads resulted in the identification of a total of 19,267 transcripts in the C. reinhardtii strain GY-D55. Th identification of novel genes/transcript isoforms has emerged as one of the major advantages of RNA-sequencing analysis. We identified a total of 4,583 novel transcript isoforms in C. reinhardtii strain GY-D55.

\section{Time-Course Transcriptome of Chlamydomonas reinhardtii Strain GY-D55 in Response to Salt Stress}

To elucidate the time-course of the transition in the gene expression of the $C$. reinhardtii strain GY-D55 under saline condition, we tracked changes in mRNA abundance by RNAseq at control and time points $(2,4,8,12,24,48,72$, and $96 \mathrm{~h}$ ) after alga cells were treated by $200 \mathrm{mM} \mathrm{NaCl}$. Principal component analysis (PCA) indicated an apparent difference in gene expression between samples under control and different time-point salt stress. Clustering analysis using whole gene expression profiles showed an apparent time-dependent similarity in the profiles of the control and $\mathrm{NaCl}$ treated samples (Figure 2A): (1) the global gene expression of control samples was most distinct from the salt stress treated time points; (2) gene expression of 2-24 h NaCl treated samples were scattered; (3) samples of salt stress treated 48, 72, and $96 \mathrm{~h}$ clustered together, indicating these stages are closer at gene expression. This result is consistent with the gene expression correlation analysis (Figure 2B) that gene expression of control samples was lowly correlated with the salt-treated samples $\left(R^{2}=0.733-0.832\right)$ and samples of late time points were highly correlated with each other $\left(R^{2}=0.945-0.963\right)$.

\section{Detection of Differentially Expressed Genes}

The relative expressions of genes in C. reinhardtii strain GYD55 under control or salt stress treatments at eight time points were evaluated based on the FPKM values. Supplementary Table 3 shows the numbers of DEGs in the control samples and $\mathrm{NaCl}$ treated samples at eight time points. A total of $9,184($ up $=4,412 /$ down $=4,772), 9,872(4,887 / 4,985), 10,901$ $(5,555 / 5,346), 9,570(4,702 / 4,868), 8,167(3,805 / 4,362), 9,877$ $(4,880 / 4,997), 8,479(4,055 / 4,424)$, and $9,766(4,846 / 4,920)$ DEGs were identified in $\mathrm{NaCl}$ treated samples at eight time points compared with the control group (Supplementary Table 3). When the transcripts were compared at each time point under the saline condition, the number of DEGs between time points was the highest for 8 vs 48 h 9,776 (4,845/4,931) and 8 vs $96 \mathrm{~h}$ $9,244(4,575 / 4,669)$, and the lowest for 72 vs 96 h $865(371 / 494)$ and $48 / \mathrm{h}$ vs $72 / \mathrm{h} 2,760(1,596 / 1,164)$, and there were little DEGs across $\mathrm{NaCl}$ treated samples among the time points of 48, 72, and $96 \mathrm{~h}$ (Supplementary Table 3). Overlapping studies found that there were 1,861/2,270 common upregulated/downregulated genes for each time point compared with control samples, and the overlapping details were shown in Figures 2C,D. Samples treated with salt for 2,8 , and $24 \mathrm{~h}$ had a relatively large number of characteristic upregulated/downregulated genes.

\section{Differentially Expressed Genes at Different Time Points and Function Enrichments}

To further provide insights into the functional transitions along with salt stress response in C. reinhardtii strain GY-D55, we clustered the 12,445 DEGs into eight clusters using the Euclidean 


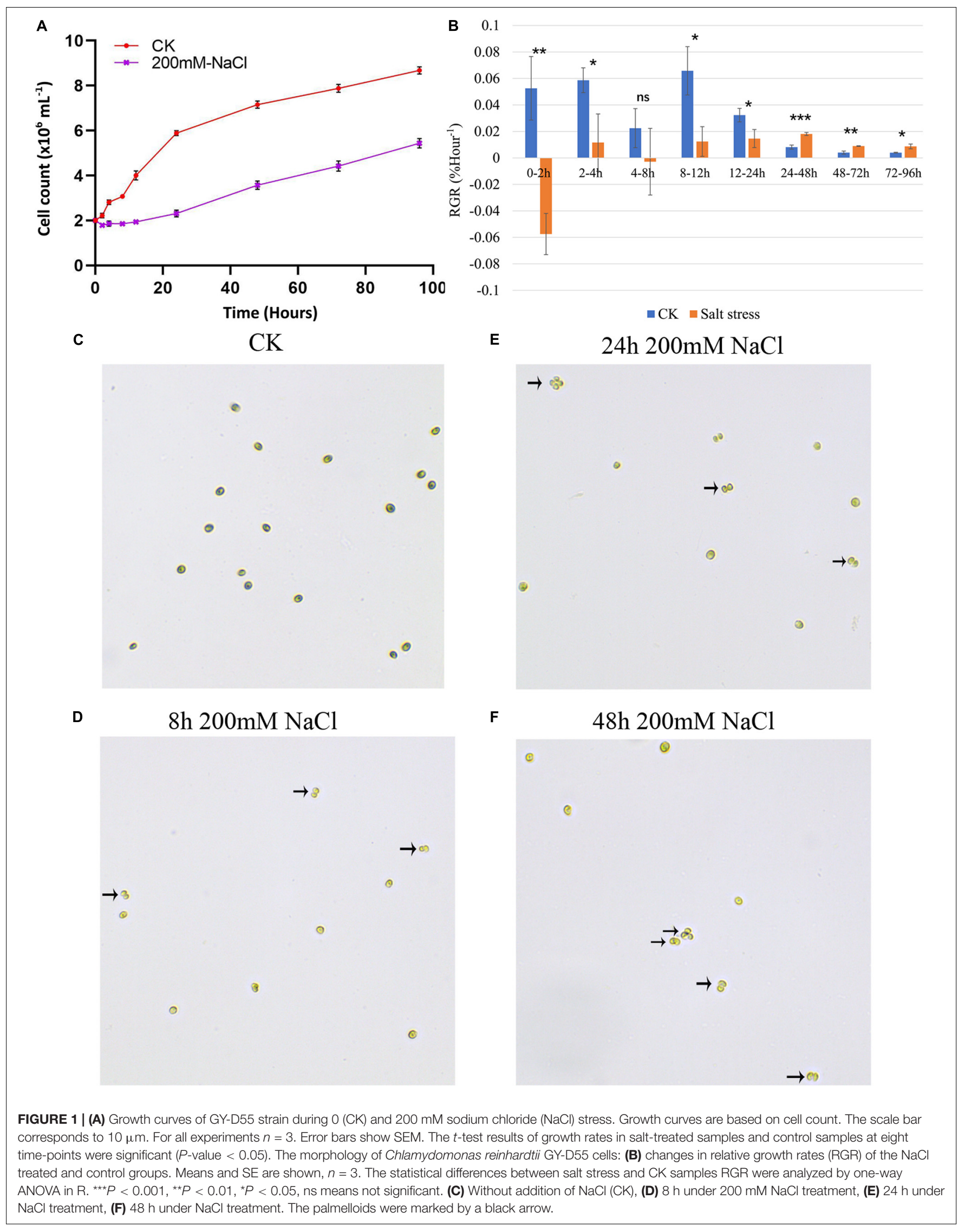




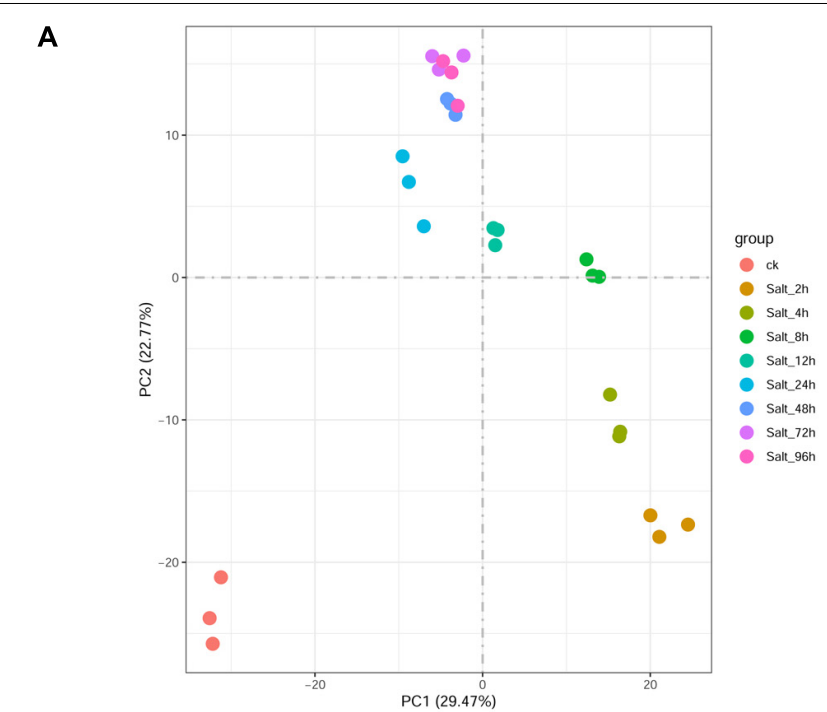

C

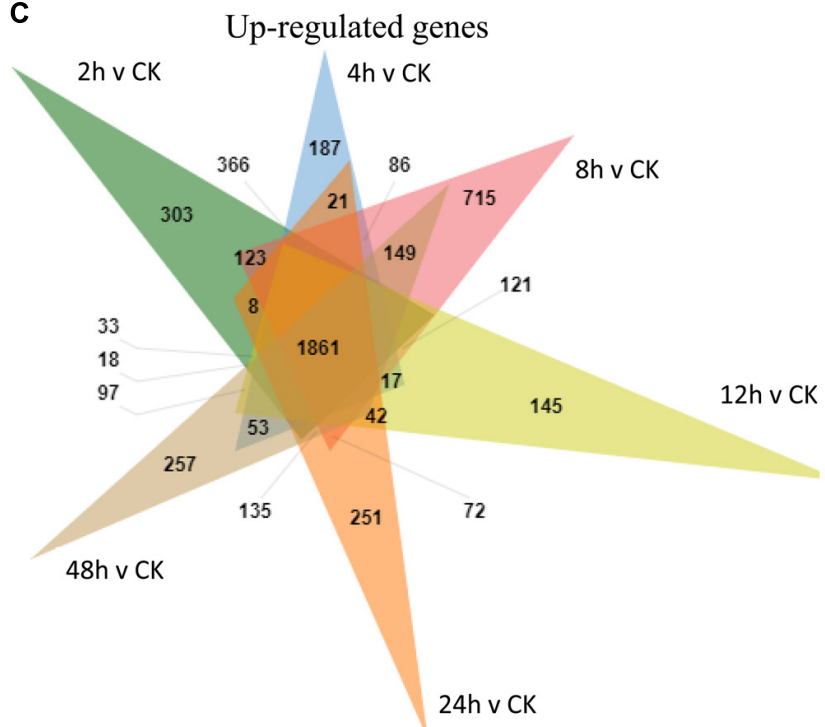

B

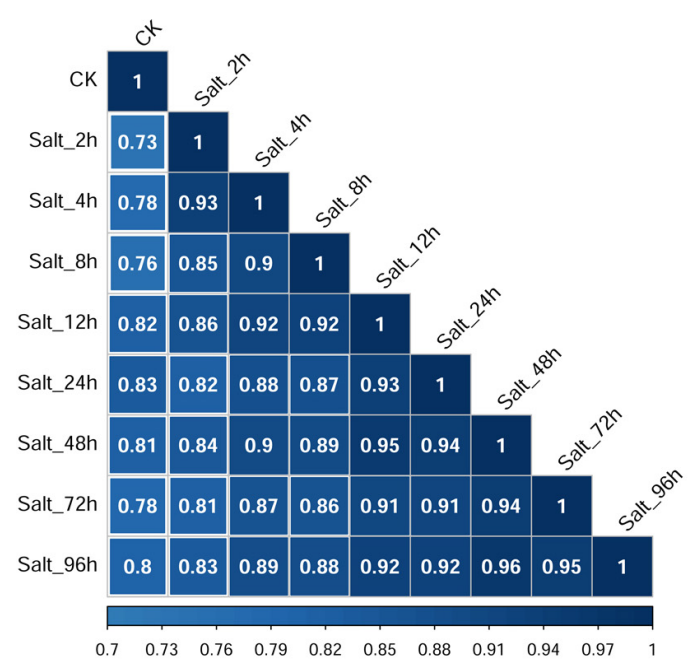

D

Down-regulated genes

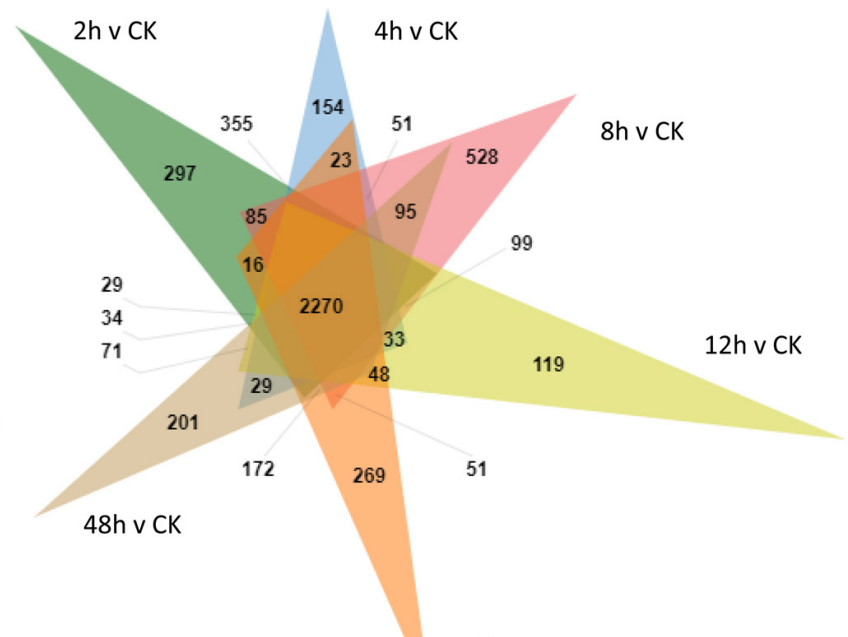

$24 \mathrm{~h} v \mathrm{CK}$

FIGURE 2 | (A) The principal component analysis (PCA) plot and (B) gene expression correlation analysis using whole gene expression profiles of GY-D55 cells in different salt stress treated time points. The PCA shows the global gene expression of control samples was most distinct from the salt stress treated time points; gene expression of 2-24 h NaCl treated samples were scattered; samples of salt stress treated 48, 72, and $96 \mathrm{~h}$ clustered together. The correlation analysis indicated that gene expression of control samples was lowly correlated with the salt-treated samples and samples of late time points were highly correlated with each other. PCA, principal component analysis. (C) The Venn analysis result of upregulated genes for each time point compared with control samples. (D) The Venn analysis result of downregulated genes for each time point compared with control samples.

distance clustering algorithm (Figure 3A and Supplementary Table 4). The GO annotation was performed to assign genes to functional categories for each cluster (Figure 3B). Genes belonging to cluster 5 (C5) were mainly expressed at 2-4 h under the salt stress, and genes in clusters 6, 7, and 8 (C6, $\mathrm{C7}$, and C8) were synchronously downregulated from 2 to $96 \mathrm{~h}$ under the saline stress. The early stage $(2-4 \mathrm{~h})$ was best represented by 3,100 expressed genes in C5 (Figures 3A,B). This cluster contained a set of genes related to "mRNA splicing, via spliceosome," "protein folding," "ER to Golgi vesicle-mediated transport," and "regulation of transcription, DNA-templated"
(Figure 3). Genes included in cluster 1 (C1) were downregulated at $2-8 \mathrm{~h}$ and participated in "carotenoid biosynthetic process," "chlorophyll biosynthetic process," and "photosystem II assembly" (Figure 3B and Supplementary Table 5). The genes in C6, C7, and C8 (1,819, 1,063, and 1,947 genes) were highly expressed in the control samples and represented by genes related to "photosynthesis, light harvesting in photosystem I," "oxidation-reduction process," and "ATP synthesis coupled proton transport” (Figure 3B and Supplementary Table 5). Inferred from the TFs data in A. thaliana, we identified a total of 113 TFs differentially expressed in salt stress responding in 


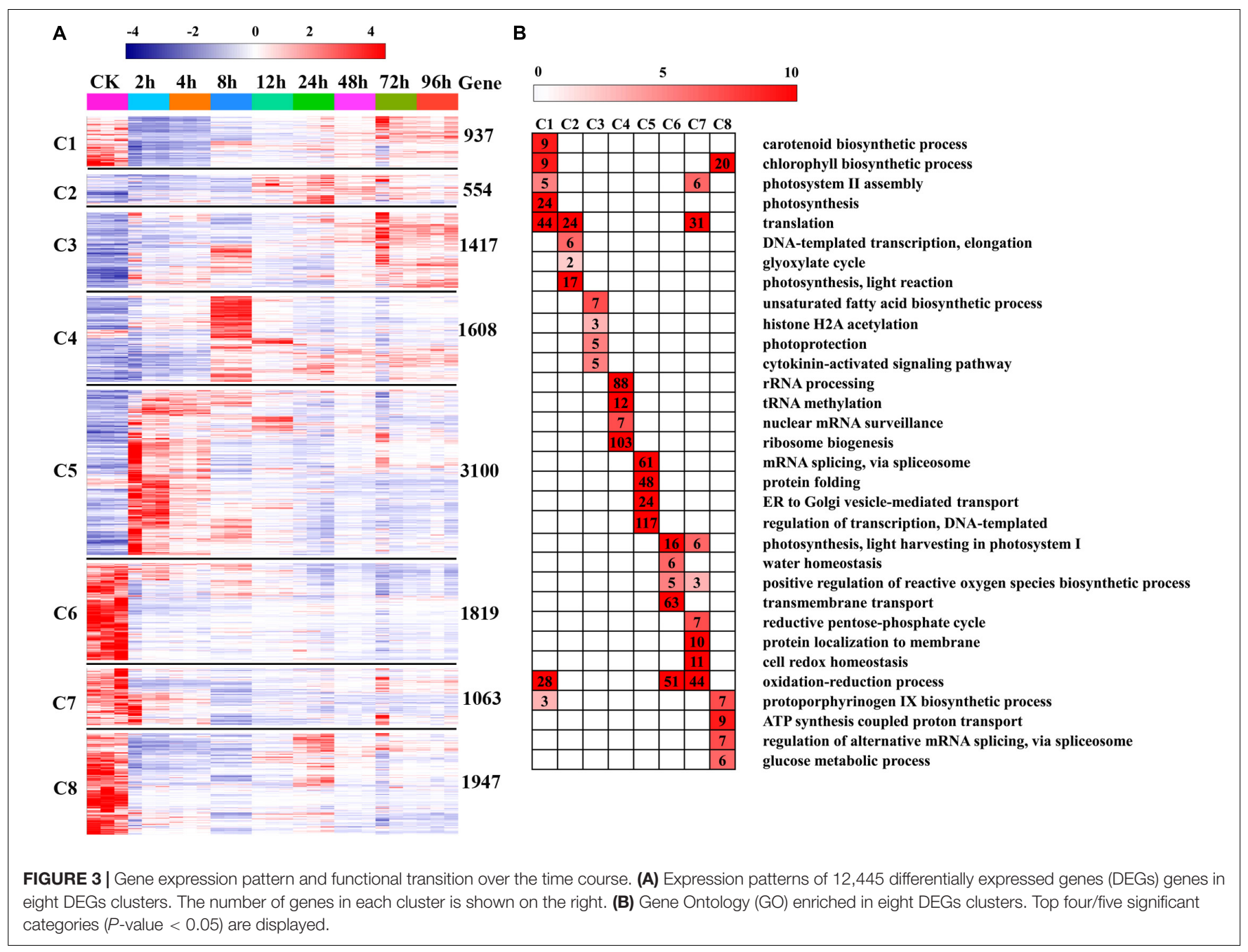

GY-D55 (Supplementary Table 6). Totals of 39.8\% (45 of 113) and $19.4 \%$ (22 of 113 ) TFs were significantly enriched in DEGs clusters C5 and C4 (Figure 4).

\section{Weighted Gene Co-expression Network Analysis Co-expression Network Construction}

A total of 18,417 genes were included in WGCNA. By sample clustering, no outliers were observed in 27 samples, thus all samples were included in the analysis. Then the soft threshold was determined by scale independence and mean connectivity analysis of modules with different power values ranging from 1 to 20 . In our study, power $=7$ was set to guarantee high scale independence (near 0.8) and low mean connectivity (near 0) (Supplementary Figure 1A). The mergeCutHeight was set as 0.25 , and a total of 44 modules were generated and displayed with different colors (Supplementary Figure 1B). All analyzed genes were included in the 44 modules (Supplementary Table 7), 4,041 and 2,299 genes were assigned to the turquoise and blue module, red and magenta module included 864 and 515 genes, 314 genes were grouped to tan module.

\section{Module-Trait Relationship Calculation}

The salt stress treated time points were collected as salt stress responding trait, and module-trait relationships were calculated according to the positive correlation between 44 modules and traits. The module trait relationship is shown in Supplementary Figure 2 and Table 1. There were five modules found positively associated with salt stress responding (Table 1). Module blue (cor $=0.592,0.391,0.435)$ was discovered associated with $2 \mathrm{~h}$ $\mathrm{NaCl}$ treated samples; the module brown ( $\mathrm{cor}=0.514,0.509$, 0.532 ) and magenta (cor $=0.384,0.417,0.451)$ were related with $8 \mathrm{~h}$; module $\tan$ (cor $=0.429,0.471,0.505$ ) were discovered associated with $12 \mathrm{~h}$ salt-treated samples; module darkturquoise (cor $=0.393,0.396,0.354$ ) was related with $24 \mathrm{~h}$. Besides, module red was found mildly associated with $2-8 \mathrm{~h}$ salt-treated samples (Supplementary Figure 2).

\section{Function Annotation of the Trait Related Modules}

The results of the GO analysis of the filtered salt stress responding related modules were shown in Table 1 and Supplementary Table 8. For salt stress 2-4 h related module blue, we identified 


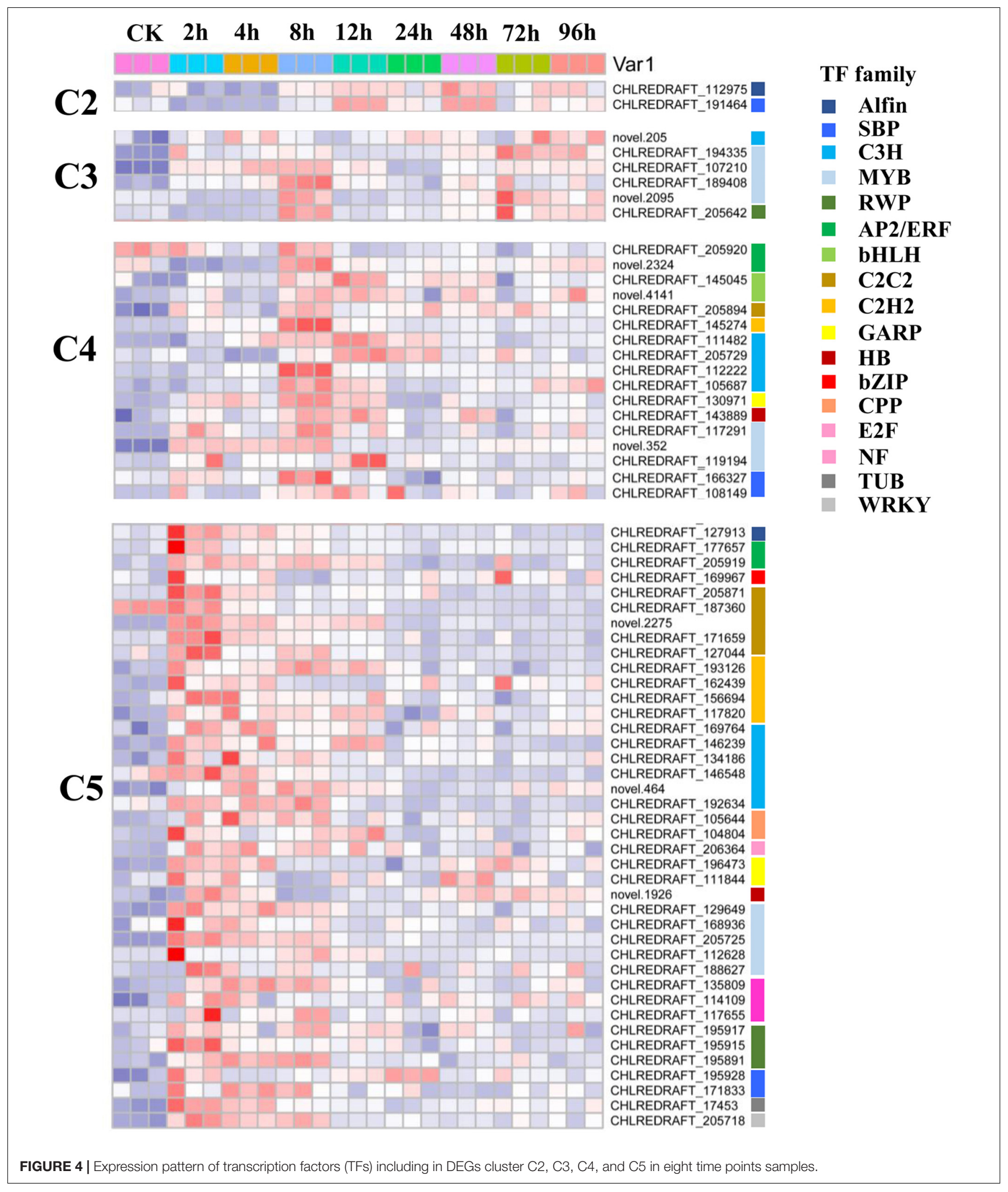

47 GO terms, including "protein phosphorylation," "signal transduction," and "regulation of DNA-templated transcription, elongation." A total of 45 terms were annotated in $8 \mathrm{~h}$ associated module magenta, including "inositol phosphate biosynthetic process," "potassium ion transport," and "auxin activated signaling pathway." For $12 \mathrm{~h}$ related module tan, we 
TABLE 1 | Module-trait relation and gene function enrichment information of genes in salt stress responding time points related modules.

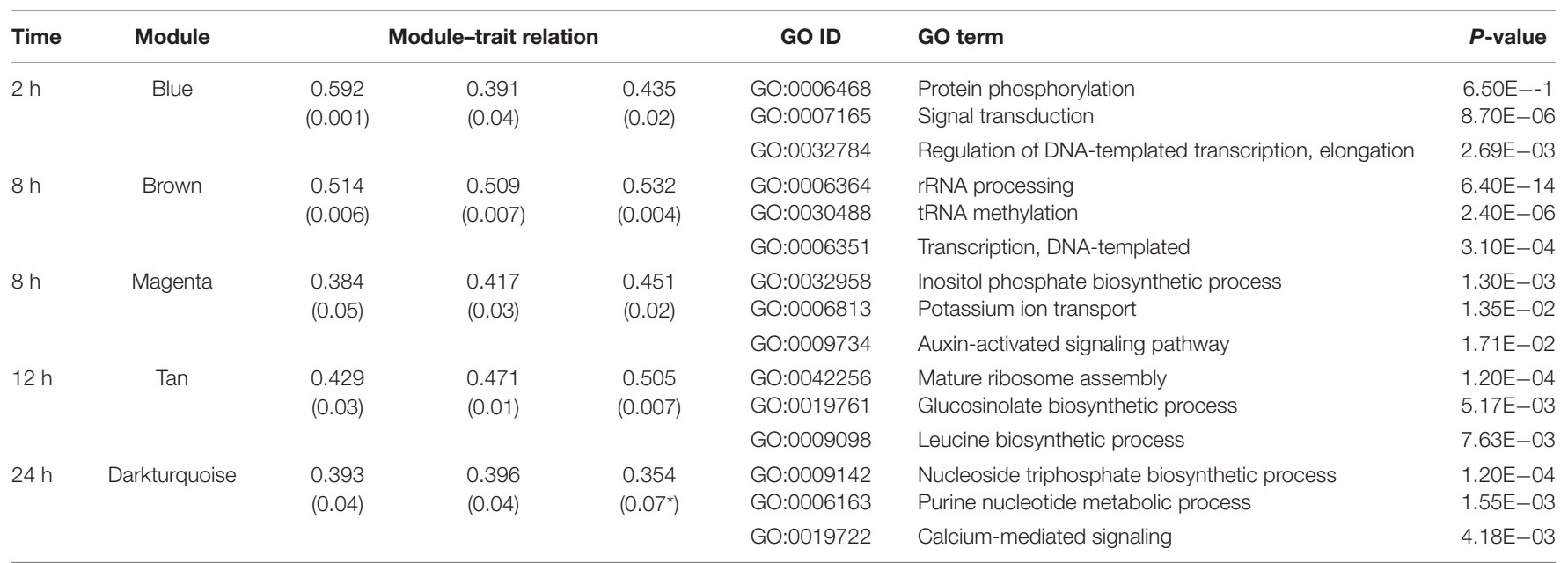

GO, Gene Ontology. *Represent the P-value $>0.05$.

identified 43 GO terms, including "mature ribosome assembly," "glucosinolate biosynthetic process," and "leucine biosynthetic process." A total of 41 terms were annotated in $48-96 \mathrm{~h}$ associated module yellow, including "cellular response to red light," "photoprotection," and "chlorophyll biosynthetic process." Besides, for salt stress 2-8 h negatively related module red, "reductive pentose phosphate cycle", "chlorophyll biosynthetic process", "oxidation-reduction process", and "photosystem II assembly" were enriched (Supplementary Table 8).

\section{Transcription Factors Regulation in Weighted Gene Co-expression Network Analysis Co-expression Module Blue}

A total of 1,192 DEGs cluster C5 genes were included in WGCNA co-expression module blue, including 17 TFs. Top 1\% co-expression links of module blue were extracted for reducing complexity. The five C5 TFs included in the top 1\% co-expression links in module blue are Alfin-CHLREDRAFT_127913, C2C2CHLREDRAFT_205871, C2C2-CHLREDRAFT_171659, MYBCHLREDRAFT_168936, and RWP-CHLREDRAFT_195915. Alfin-CHLREDRAFT_127913, C2C2-CHLREDRAFT_205871, and MYB-CHLREDRAFT_168936 were predicted to interact with 173, 72, and 6 genes, respectively, some of their potential target genes are overlapped.

\section{Real-Time Quantitative PCR Validation}

To verify the RNA-seq results, an alternative strategy was selected for the upregulated genes. In total, eight genes were selected for validation by qRT-PCR using the same RNA samples that were used for RNA-sequencing (Supplementary Figure 3). The primers' sequences were shown in Supplementary Table 9. In most cases, the gene expression trends were similar between these two methods, Pearson's correlation of gene expression between qRT-PCR analysis and RNA-Seq data is cor $=0.765, P$-_value $<3.464 \mathrm{e}^{-07}$; the result is shown in Supplementary Figure 3.

\section{DISCUSSION}

Existing research on $C$. reinhardtii shows that at the morphological level, salt stress of 150 or $200 \mathrm{mM} \mathrm{NaCl}$ results in flagellar resorption, decreased cell size, impaired growth, and palmelloid morphology (Neelam and Subramanyam, 2013; Khona et al., 2016). In the study published by Khona et al. (2016), the immediate death of $C$. reinhardtii cells was observed with 350 and $500 \mathrm{mM} \mathrm{NaCl}$. We have tested the $300 \mathrm{M} \mathrm{NaCl}$ treatment on GY-D55 cells, a large number of cells death was observed with this concentration, which suggested the $\mathrm{NaCl}$ tolerance limits of green algae C. reinhardtii strain GY-D55. In the present study, the growth rate of $C$. reinhardtii strain GY-D55 was significantly impaired in the presence of $200 \mathrm{mM}$ $\mathrm{NaCl}$ (Figure 1A), especially at early time points (2-12 h). Moreover, we characterized the formation of palmelloids in GY-D55 cells under salt stress. In land plants, cell-to-cell adhesion is regulated via a middle lamella mainly consisting of pectic polysaccharides. As a Gal oxidase, $R U B Y$ enhances pectin cohesion within the middle lamella of seed coat mucilage in A. thaliana (Sola et al., 2019). UDP-glucose pyrophosphorylase UGP1/2 is an enzyme playing a critical role in the metabolism of UDP-glucose, a precursor for the synthesis of carbohydrate cell wall components, including cellulose and callose (Klopffleisch et al., 2011). Besides, UDP-glucosyltransferase UGT-80 involves in the carbohydrate metabolic process. The upregulation of RUBY (CHLREDRAFT_196816) at 2-48 h, UGT-80 (CHLREDRAFT_154976) at 2-12 h, and UGP2 (CHLREDRAFT_157049) at 2-4 h in GY-D55 cells suggested the function of these genes in cell wall carbohydrates of palmelloid formation during the early stage of salt stress in microalgae.

Genes in DEG clusters C1, C6, and C8 were significantly downregulated at 2-4 $\mathrm{h}$ (Figure 3A), and the functional enrichment analyses of these genes suggested that short-term salt stress impaired oxidation-reduction, protein modification, water homeostasis, and chlorophyll biosynthesis of GY-D55 cells, which was consistent with the previous study. In the published 
studies, the cell growth and photosynthetic activity in several strains of $C$. reinhardtii recover over $24 \mathrm{~h}$. In the present study, clusters C5, C4, C2, and C3 contained genes mainly expressed at 2-4, 8, 12, and $24 \mathrm{~h}$, respectively (Figure 3A). We predicted the dynamic responding mechanisms of these clusters to salt stress in GY-D55 cells. The algal cells promoted the transcriptional regulation and protein folding to deal with protein synthesis/folding and other impairments caused by saline stress at 2-4 h. In $8 \mathrm{~h}, \mathrm{GY}-\mathrm{D} 55$ cells mainly regulated the synthesis and regulation of rRNA and tRNA to deal with salt stress. At $12 \mathrm{~h}$, GY-D55 cells promoted transcription and translation and improved the expressions of photosynthetic genes to deal with the damage of photosynthesis. Light protection and cytokinin signaling pathways were activated in $24 \mathrm{~h}$ to promote the recovery of photosynthesis and regulate transcription.

Chlamydomonas response to abiotic stresses, including salt stress, has been investigated at both the proteomic and transcriptomic levels. Quantitative proteomic comparison of salt stress in C. reinhardtii strain CC-4325 and the snow alga C. nivalis reveals that salt stress enhances the accumulation of fatty acids in C. nivalis (Hounslow et al., 2021), C. reinhardtii does not have this response. The transcriptome analysis was used to investigate the molecular and genetic mechanisms of salt stress responses in C. reinhardtii strain GY-D55 after $24 \mathrm{~h}$ of acclimation to $200 \mathrm{mM} \mathrm{NaCl}$ (Wang et al., 2018). We compared several key genes'/proteins' changes during salt stress in the abovementioned studies and current work, and these genes/proteins are related to stress tolerance, photosynthesis, carbohydrate metabolism, and lipid metabolism (Table 2). In most cases, the gene expression trends were similar among the three studies. CC4325 cells showed a higher abundance of HSP 70B after being treated with salt stress for $11 \mathrm{~h}$, and the coding gene of HSP70B (CHLREDRAFT_126835) was upregulated at 8-96 h compared with control samples in GY-D55 cells. HSP70B has previously been shown to increase under salinity stress in salt-adapted C. reinhardtii cells and involve in the photosystem II damage (Sithtisarn et al., 2017) and repair process in D. salina (Goyal, 2007). These results suggest that it plays a similar role in salinity response in strain CC-4325 and GY-D55. Pyrroline-5-carboxylate synthetase (P5CS) catalyzes the first step in proline (Perrineau et al., 2014; Zhu, 2016) biosynthesis from glutamate, and we found that P5CS2 (CHLREDRAFT_130812) was upregulated at $24 \mathrm{~h}$ in the analysis performed by Wang et al. (2018) and this work, suggesting that P5CS2 regulated proline synthesis in modulating the salt stress tolerance in GY-D55 cells. In both transcriptomic analyses, the coding gene of glutathione $\mathrm{S}$ transferase (CHLREDRAFT_195543) was upregulated under the saline stress in GY-D55 cells, while many genes of the ROSscavenging system were downregulated.

Photosynthetic activity in C. reinhardtii is initially suppressed in the presence of $200 \mathrm{mM}$ salt, while it recovers over $24 \mathrm{~h}$ (Crespo, 2012; Erickson et al., 2015). The CC-4325 and GY-D55 cells show decreased expressions of photosynthetic proteins/genes. Photosystem (PS)I reaction centers and lightharvesting protein were largely decreased, including photosystem I reaction center subunit III (CHLREDRAFT_130914) and light-harvesting chlorophyll- $a / b$ protein of photosystem I
(CHLREDRAFT_136294). Although the expressions of several PSI- and PSII-related genes slightly recovered after $24 \mathrm{~h}$ in GYD55 cells (Supplementary Figure 4). PSI has a slower recovery than PSII once damage occurs. These impairments of PSI indicated longer-term damage to the photosynthetic apparatus in CC-4325 and GY-D55 cells. Oxygen evolving enhancer proteins of PSII remained unchanged at $11 \mathrm{~h}$ in CC-4325 cells, whilst it was decreased at $2 \mathrm{~h}$ in GY-D55 cells. This result indicated that PSII impairments occurred in GY-D55 cells early than CC4325 cells. For alga, starch remobilization is important for starch storage to lipid storage and production of glycerol. UDP-glucose 6-dehydrogenase is an enzyme that catalyzes starch into glucose for use in glycolysis (Hounslow et al., 2021). Salt exposure caused a higher abundance of this protein over time in CC4325 cells, while its coding gene (CHLREDRAFT_185081) was upregulated at a late stage (72-96 h) in this study and not altered in the study of Wang et al. (2018) in GY-D55 cells. Algae can divert cellular resources toward lipid accumulation under salt stress (Hounslow et al., 2021). UDP-sulfoquinovose synthase is involved in glycerolipid metabolism and thylakoid membrane sulfoquinovosyl diacylglycerol (SQDG) metabolism in algae. We found that the abundance of SQDG (CHLRE-DRAFT_27658) was reduced in GY-D55 cells at $2-8 \mathrm{~h}$ in our study, and it is downregulated at $24 \mathrm{~h}$ in the study of Wang et al. (2018), while it was not changed in CC-4325 cells. Although the depressed expression of $S Q D G$ provides a potential mechanism for a key difference between alga's fatty acid responses, this strain did not display the lipid accumulation response to salt stress with the downregulation of fatty acid desaturase. This finding helped elucidate that what caused salt stress was a lipid trigger, but not C. reinhardtii strain GY-D55.

Glycerol is an effective compatible solute that is produced by most salt-sensitive algal species under high saline stress (Goyal, 2007; Meijer et al., 2017; Hounslow et al., 2021). In the present study, the time course expressions of key genes were mapped into the schematic diagram of starch metabolism, glycerol production, and lipid synthesis (Figure 5). In the first step of glycolysis, starch is converted to glucose by alpha-amylase (AMY1, CHLREDRAFT_173725) (Shetty et al., 2019). The product of glycolysis is converted to glyceraldehyde-3-phosphate (GA3P) and then converted to dihydroxyacetone-phosphate (DHAP) by triosephosphate isomerase (Shetty et al., 2019) (TIM, CHLREDRAFT_26265). These two genes are mainly expressed at 2-4 h under salt stress in GY-D55 cells. Then, DHAP is converted to glycerol-3-phosphate (G3P) by glycerol-3-phosphate dehydrogenase (G3PDH). There are five isoforms of $\mathrm{G} 3 \mathrm{PDH}$ enzyme in $C$. reinhardtii (Shetty et al., 2019). In the present study, two genes (GPD1, CHLREDRAFT_94229; GPDHp, CHLREDRAFT_146945) showed an over-expression pattern at 2-4 h under salt stress, and one gene (GPDHp, CHLREDRAFT_146946) was mainly expressed at 8-12 h. The homolog of glycerol-3-phosphatase (GS1, CHLREDRAFT_184987) catalyzes the formation of glycerol in higher plants (Singh et al., 2016; Shetty et al., 2019), and the homolog of this gene is upregulated at 4,72 , and $96 \mathrm{~h}$ in C. reinhardtii. The over-expression of key genes at $2-8 \mathrm{~h}$ under salt stress indicated that GY-D55 cells rapidly 
TABLE 2 | Comparisons of gene/protein changes during salt stress in published studies and this study.

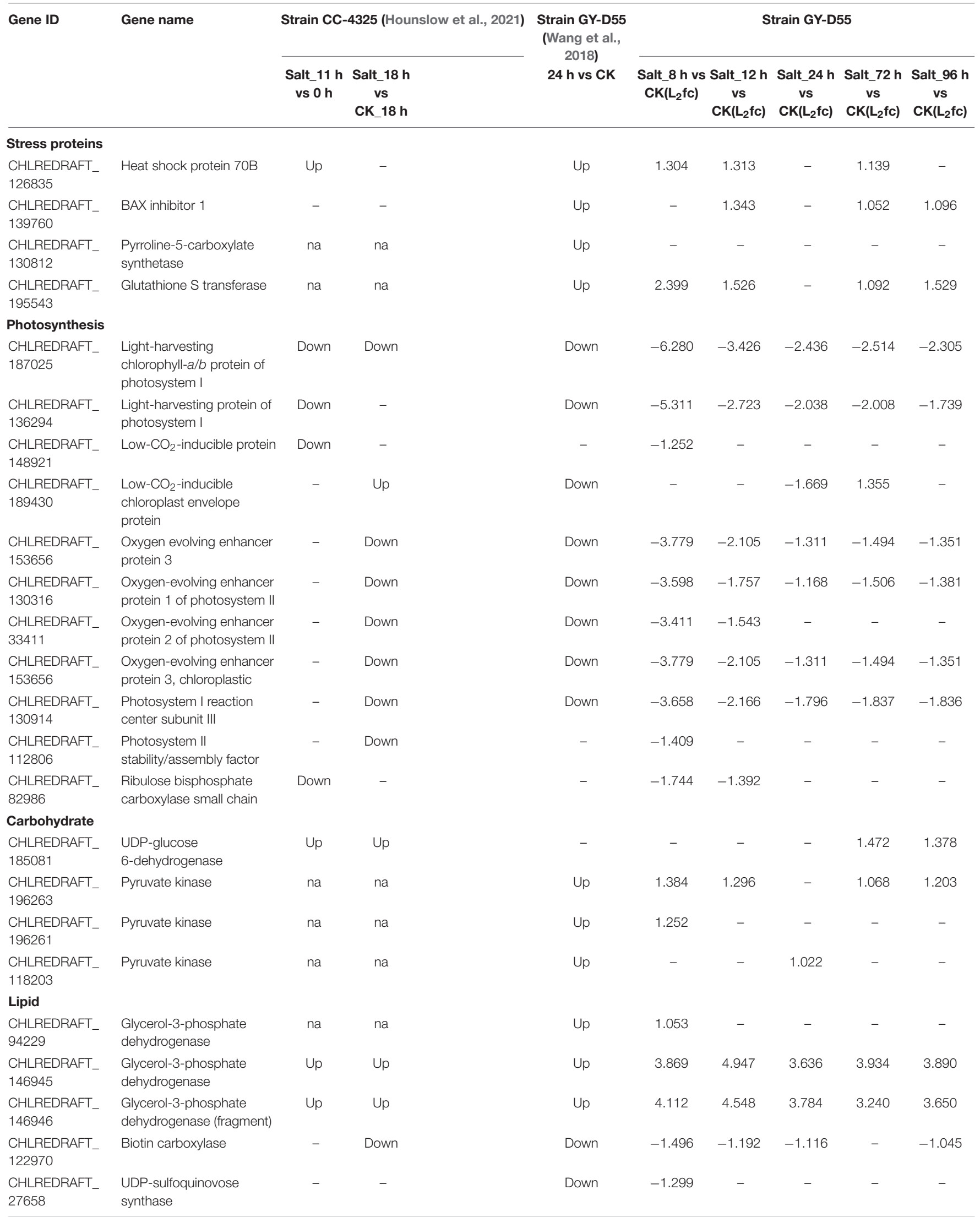

"-" means the difference was not significant. $L_{2} f c$, Log 2 fold change; Up, upregulated; Down, downregulated; na, not mentioned. 


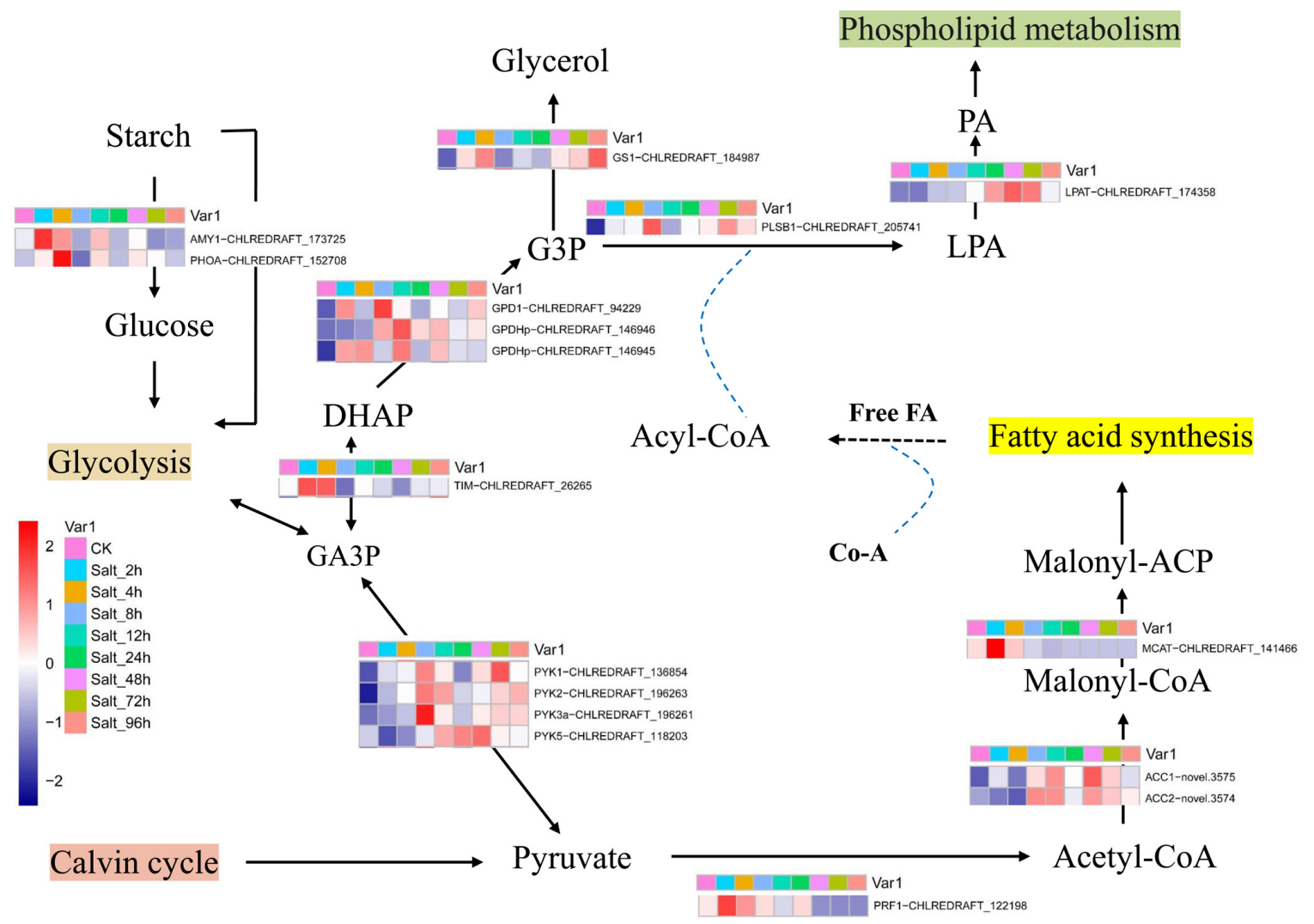

FIGURE 5 | The schematic diagram of starch metabolism, glycerol production, and lipid synthesis. GA3P, glyceraldehyde3-phosphate; G3P, glycerol-3-phosphate; DHAP, dihydroxyacetone phosphate; LPA, lysophosphatidic acid; PA, phosphatidic acid. Mean FPKM values of the three samples in each time point were used as representative of genes' expression value in eight salt-treated time points and control conditions.

accumulated glycerol in the early stage of salt stress to cope with osmotic stress. There is an interplay between photosynthetic rate and the limited ability of the organism to utilize/export/store the photosynthetically fixed carbon. In the present study, photosynthesis was significantly inhibited before $24 \mathrm{~h}$, which might be related to the accumulation of glycerol in the cells. Releasing large quantities of photosynthetic products leads to an increase in photosynthetic rates in plants. We speculated that GY-D55 cells leaked glycerol into the surrounding environment after $24 \mathrm{~h}$ to promote photosynthetic efficiency.

Transcription factor-encoding genes contribute to the diversity and evolution of plants. Identification of the TFs is essential in manipulating the regulatory network for abiotic stress in response to the target molecules (Lehti-Shiu et al., 2017). Inferred from the TF data in A. thaliana, we identified a total of 113 differentially expressed TFs in response to salt stress in GY-D55 (Supplementary Table 6). Moreover, 39.8 and 19.4\% TFs were significantly enriched in DEG clusters C5 and C4, respectively, reflecting the important role of TFs in the early stage of salt stress in C. reinhardtii strain GY-D55 (Figure 4 and Supplementary Table 6). Several members from the bZIP, $\mathrm{C} 3 \mathrm{H}$, Dof, and MYB TF families were differentially expressed throughout the entire time course in salt and cold stresses. In the present study, eight/seven C3H TFs were upregulated at $2-4 / 8 \mathrm{~h}$, respectively, indicating their functions in salt stress regulation. A total of five MYB TFs were clustered in C5, including a homolog of MYB88 (CHLREDRAFT_205725). In A. thaliana, MYB88 participates in the stomatal development in abiotic stress responses and the regulation of cell cycle genes (Xie et al., 2010). Chlamydomonas is a single-cell green alga, and there are no complex vegetative structures, such as leaves. However, TFs in plants have a higher retention rate after duplication compared with other genes (Lehti-Shiu et al., 2015; Thiriet-Rupert et al., 2016). Additionally, genes functionally related to stress responses tend to undergo a more intense duplication process (RianoPachon et al., 2008; Wu et al., 2015, 2021). Therefore, in higher plants and algae, the functions of genes in the same family or homologous group may be different, while they may participate in stress response conservatively.

Genes involved in related BPs tend to be co-expressed and clustered as functional modules (Ruprecht et al., 2017), which can help identify how the interplay between interconnected genes accomplishes specific biological functions. In the present study, "protein phosphorylation," "signal transduction," and "regulation of DNA-templated transcription, elongation" were top enriched BPs in the 2-4 h related module blue. Current evidence suggested that endoplasmic reticulum (ER) stress occurred at early time points $(2-8 \mathrm{~h})$ 


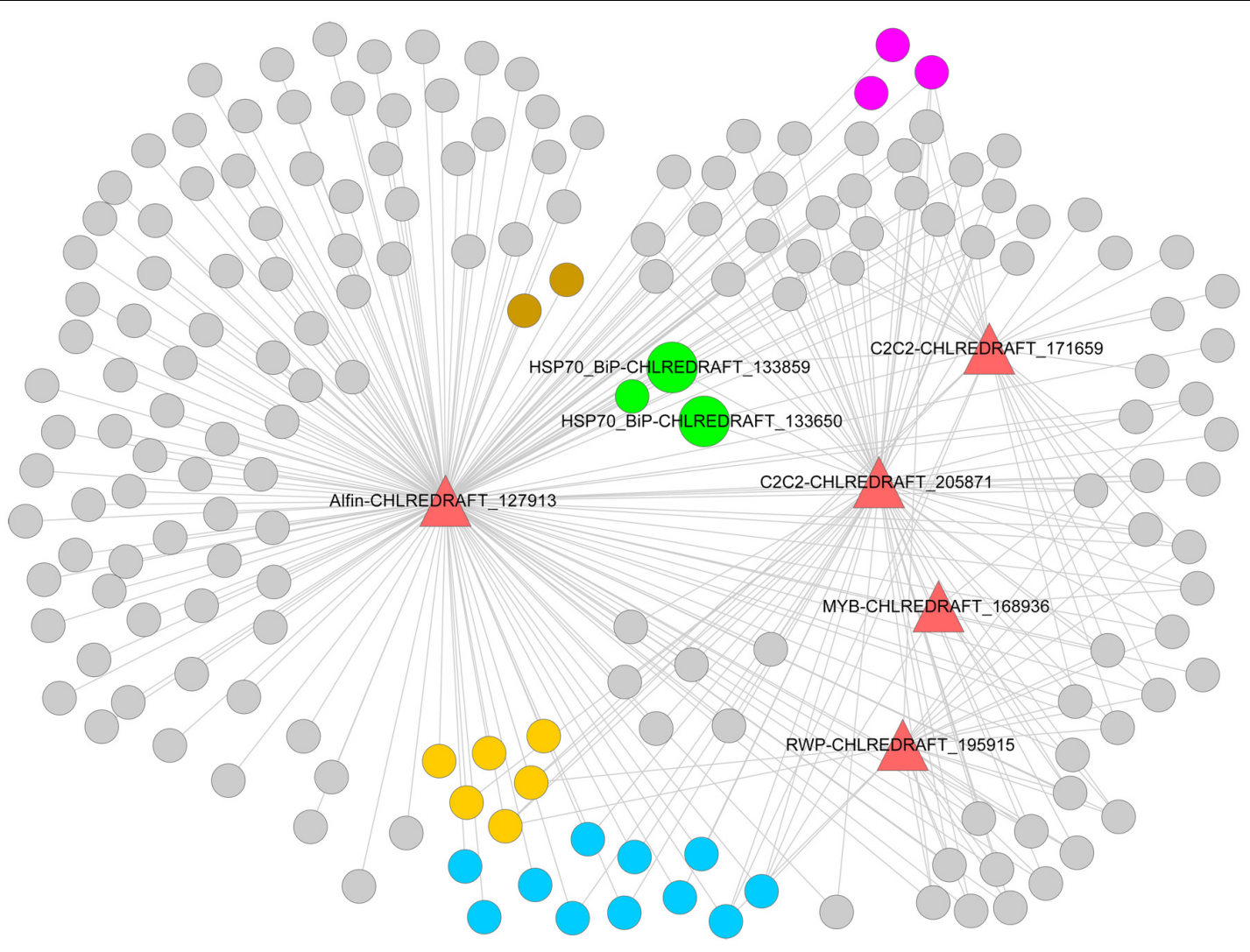

Node shape

Node color

$\bigcup_{\text {Others }}$ Transcriptional factor

Transcriptional factor

Water transport

Others

Establishment of protein localization

Endoplasmic reticulum unfolded protein response

Regulation of cell cycle

Cellulose biosynthetic process

FIGURE 6 | Five TFs regulating genes in co-expression module blue. This module was predicted to regulate endoplasmic reticulum (ER) stress at early time points. TFs (Alfin-CHLREDRAFT_127913, C2C2-CHLREDRAFT_205871, and C2C2-CHLREDRAF__171659) regulating 11 genes of "establishment of protein localization" and three genes of "endoplasmic reticulum unfolded protein response," including ER stress key gene BiP (CHLREDRAFT_133650 and CHLREDRAFT_133859).

in GY-D55 cells. As shown in Figure 6, in module blue, Alfin-CHLREDRAFT_127913, C2C2-CHLREDRAFT_205871, and C2C2-CHLREDRAFT_171659 regulated 11 genes of "establishment of protein localization" and three genes of "endoplasmic reticulum unfolded protein response," including ER stress key gene BiP (CHLREDRAFT_133650 and CHLREDRAFT_133859). As a member of the HSP70 family, $B i P$ resides within the lumen of the ER, and it helps the folding and assembly of newly synthesized proteins as they are translocated into the ER and can also bind to misfolded, underglycosylated, or unassembled proteins (Crespo, 2012). Several members of Alfin-like TFs were upregulated in response to different abiotic stresses in Brassica oleracea. The role of Alfin-like TFs in enhancing salt stress and drought resistance is well-known when it is over-expressed in land plants roots. These results suggest the important role of the TFs of the Alfin and $\mathrm{C} 2 \mathrm{C} 2$ families in salt stress in GY-D55 cells, especially in ER stress. In C. reinhardtii, most of the published studies have focused on the mechanisms of bZIP and MYB TFs in salt stress, while the TFs of other families are rarely reported (Ji et al., 2018). The present work provided a valuable foundation for TFs, benefiting the exploration of the regulatory network in algal abiotic stress.

\section{CONCLUSION}

Here, we reported a comprehensive temporal investigation of transcriptomes using data at eight different time points, from early stage $(2 \mathrm{~h})$ to late stage (up to $96 \mathrm{~h}$ ) in C. reinhardtii GYD55 cells. A total of 12,445 genes were detected as DEGs. There were 1,861/2,270 common upregulated/downregulated genes for each time point compared with control samples. Samples treated 
with salt for 2,8 , and $24 \mathrm{~h}$ had a relatively large number of characteristic upregulated/downregulated genes. Short-time exposure to salt stress impaired oxidation reduction, protein synthesis and modification, and photosynthesis. The algal cells promoted transcriptional regulation and protein folding to deal with protein synthesis/modification impairments and rapidly accumulated glycerol in the early stage $(2-4 \mathrm{~h})$ to cope with osmotic stress. At 12 and 24 h, GY-D55 cells showed increased expressions of signaling and photosynthetic genes to deal with the damage of photosynthesis. The co-expression module blue was predicted to regulate ER stress at early time points. In addition, we identified a total of $113 \mathrm{TFs}$ and predicted the potential roles of Alfin, C2C2, and MYB family TFs in algal salt stress responses.

\section{DATA AVAILABILITY STATEMENT}

The original contributions presented in the study are publicly available. This data can be found here: National Center for Biotechnology Information (NCBI) BioProject database under accession number PRJNA770825 and the Gene Expression Omnibus (GEO) database, GSE191218.

\section{AUTHOR CONTRIBUTIONS}

L-YZ and S-JF designed the experiments. L-YZ, Z-TX, and L-QC carried out the experiment and analyzed the data. L-YZ and Z-TX wrote the first draft of the manuscript. L-YZ, X-JZ, and S-JF supervised and completed the writing. All authors revised and approved the final manuscript.

\section{FUNDING}

This work was supported by the National Natural Science Foundation of China (31800185) and the A Project of Shandong Province Higher Educational Science and Technology Program (J18KA147).

\section{SUPPLEMENTARY MATERIAL}

The Supplementary Material for this article can be found online at: https://www.frontiersin.org/articles/10.3389/fpls.2022. 828321/full\#supplementary-material

\section{REFERENCES}

Ahmad, I., and Hellebust, J. A. (1984). Osmoregulation in the extremely euryhaline marine micro-alga Chlorella autotrophica. Plant Physiol. 74, 1010-1015. doi: 10.1104/pp.74.4.1010

Bazzani, E., Lauritano, C., Mangoni, O., Bolinesi, F., and Saggiomo, M. (2021). Chlamydomonas responses to salinity stress and possible biotechnological exploitation. J. Mar. Sci. Eng. 9:1242. doi: 10.3390/jmse9111242

Breker, M., Lieberman, K., Tulin, F., and Cross, F. R. (2016). High-throughput robotically assisted isolation of temperature-sensitive lethal mutants in Chlamydomonas reinhardtii. J. Vis. Exp. 118:e54831. doi: 10.3791/54831
Supplementary Figure 1 | Construction of a weighted gene co-expression network. (A) Scale independence and (B) mean connectivity analysis. (C) Cluster dendrogram among modules.

Supplementary Figure 2 | The salt stress treated time points expressed genes weighted gene co-expression network analysis (WGCNA) module-trait relationship. WGCNA, weighted gene co-expression network analysis.

Supplementary Figure 3 | Validation of RNA-Sequencing (RNA-seq) data. Verification of the expression level of the selected eight differentially expressed genes (DEGs) from RNA-Seq data through RT-qPCR: (A) CHLREDRAFT_130812, (B) CHLREDRAFT_196816, (C) CHLREDRAFT_205725, (D)

CHLREDRAFT_205718, (E) CHLREDRAFT_27658, (F) CHLREDRAFT_33411, (G) CHLREDRAFT_190083, (H) CHLREDRAFT_192806. Error bars indicate the standard error as mean $+\mathrm{SD}$. The $x$-axis represents the relative expression level, and the $y$-axis represents $\mathrm{NaCl}$ treated time points. The statistical differences between salt treated and control samples were analyzed by one-way ANOVA. A total of 2, 12, 24, and $48 \mathrm{~h}$ were used as representative of eight time points in the RT-qPCR analysis. ${ }^{* * * *} P<0.0001,{ }^{* * *} P<0.001,{ }^{* *} P<0.01,{ }^{*} P<0.05$, ns means not significant. (I) Correlation coefficient of gene expression between qRT-PCR analysis and RNA-Seq data.

Supplementary Figure 4 | The Kyoto Encyclopedia of Genes and Genomes (KEGG) pathways (ko00195) "photosynthesis" mapped with downregulated genes at different time points. The downregulated genes are marked by green color. The black solid line with a black arrow means molecular interaction or relation; the black dash line with a black arrow means indirect link or unknown reaction; the red dash line with a red arrow stands for the light quanta. The heatmaps of gene clusters were shown separately. A red color indicates that the gene is highly expressed under the corresponding treatment.

Supplementary Table 1 | The t-test analysis result of the growth ratio between cells under control and saline condition.

Supplementary Table 2 | Summary of mapping transcriptome reads to the reference sequence.

Supplementary Table 3 | The differentially expressed gene number between the adjacent time point of salt stress responding in Chlamydomonas reinhardtii strain GY-D55.

Supplementary Table 4 | The 12,445 differentially expressed genes in the eight clusters.

Supplementary Table 5 | The Gene Ontology (GO) enrichment results of the eight differentially expressed gene clusters.

Supplementary Table 6 | Summary of the differentially expressed transcription factors.

Supplementary Table 7 | Gene number and edge number of 44 WGCNA modules.

Supplementary Table 8 | The GO enrichment results of 44 WGCNA modules' members.

Supplementary Table 9 | Information of the quantitative real time PCR (qRT-PCR) primers.

Chen, X., Tian, D., Kong, X., Chen, Q., Abd Allah, E. F., Hu, X., et al. (2016). The role of nitric oxide signalling in response to salt stress in Chlamydomonas reinhardtii. Planta 244, 651-669. doi: 10.1007/s00425-016-2528-0

Crespo, J. L. (2012). BiP links TOR signaling to ER stress in Chlamydomonas. Plant Signal. Behav. 7, 273-275. doi: 10.4161/psb.18767

Erickson, E., Wakao, S., and Niyogi, K. K. (2015). Light stress and photoprotection in Chlamydomonas reinhardtii. Plant J. 82, 449-465. doi: 10.1111/tpj.12825

Foflonker, F., Price, D. C., Qiu, H., Palenik, B., Wang, S., and Bhattacharya, D. (2014). Genome of the halotolerant green alga Picochlorum sp. reveals strategies for thriving under fluctuating environmental conditions. Environ. Microbiol. 17, 412-426. doi: 10.1111/1462-2920.12541 
Funck, D., Baumgarten, L., Stift, M., von Wiren, N., and Schonemann, L. (2020). Differential Contribution of P5CS Isoforms to Stress Tolerance in Arabidopsis. Front. Plant Sci 11:565134. doi: 10.3389/fpls.2020.565134

Gammoudi, S., Dahmen-Ben Moussa, I., Annabi-Trabelsi, N., Ayadi, H., and Guermazi, W. (2021). "Antioxidant properties of metabolites from new extremophiles microalgal strain (Southern, Tunisia)" in AntioxidantsBenefits, Sources, Mechanisms of Action. ed. V. Waisundara (Rijeka: IntechOpen Press).

Gierz, S. L., Foret, S., and Leggat, W. (2017). Transcriptomic analysis of thermally stressed Symbiodinium reveals differential expression of stress and metabolism genes. Front. Plant Sci. 8:271. doi: 10.3389/fpls.2017.00271

Gong, Z., Xiong, L., Shi, H., Yang, S., Herrera-Estrella, L. R., Xu, G., et al. (2020). Plant abiotic stress response and nutrient use efficiency. Sci China Life Sci 63, 635-674. doi: 10.1007/s11427-020-1683-x

Goyal, A. (2007). Osmoregulation in Dunaliella, Part II: photosynthesis and starch contribute carbon for glycerol synthesis during a salt stress in Dunaliella tertiolecta. Plant Physiol. Biochem. 45, 705-710. doi: 10.1016/j.plaphy.2007.05. 009

Grant, W. D., Gemmel, R. T., and McGenity, T. J. (1998). "Halophiles" in Extremophiles: microbial Life in Extreme Environments. eds K. Horikoshi and W. D. Grant (Hoboken: Wiley Press). 93-132.

Grossman, A. (2000). Acclimation of Chlamydomonas reinhardtii to its nutrient environment. Protist 151, 201-224. doi: 10.1078/1434-4610-00020

Hang, L. T., Mori, K., Tanaka, Y., Morikawa, M., and Toyama, T. (2020). Enhanced lipid productivity of Chlamydomonas reinhardtii with combination of $\mathrm{NaCl}$ and $\mathrm{CaCl} 2$ stresses. Bioprocess Biosyst Eng 43, 971-980. doi: 10.1007/s00449020-02293-w

Hanikenne, M. (2003). Chlamydomonas reinhardtii as a eukaryotic photosynthetic model for studies of heavy metal homeostasis and tolerance. New Phytol. 159, 331-340. doi: 10.1046/j.1469-8137.2003.00788.x

Ho, S. H., Nakanishi, A., Kato, Y., Yamasaki, H., Chang, J. S., Misawa, N., et al. (2017). Dynamic metabolic profiling together with transcription analysis reveals salinity-induced starch-to-lipid biosynthesis in alga Chlamydomonas sp. JSC4. Sci. Rep. 7:45471. doi: 10.1038/srep45471

Hounslow, E., Evans, C. A., Pandhal, J., Sydney, T., Couto, N., Pham, T. K., et al. (2021). Quantitative proteomic comparison of salt stress in Chlamydomonas reinhardtii and the snow alga Chlamydomonas nivalis reveals mechanisms for salt-triggered fatty acid accumulation via reallocation of carbon resources. Biotechnol. Biofuels 14:121. doi: 10.1186/s13068-021-01970-6

Husic, H. D., and Quigley, E. A. (1990). Salt-induced dissociation of carbonic anhydrase from intact cells of Chlamydomonas reinhardtii. Plant Physiol. 94, 380-383. doi: 10.1104/pp.94.1.380

James, G. O., Hocart, C. H., Hillier, W., Price, G. D., and Djordjevic, M. A. (2013). Temperature modulation of fatty acid profiles for biofuel production in nitrogen deprived Chlamydomonas reinhardtii. Bioresour. Technol. 127, 441-447. doi: 10.1016/j.biortech.2012.09.090

Ji, C., Mao, X., Hao, J., Wang, X., Xue, J., Cui, H., et al. (2018). Analysis of bZIP transcription factor family and their expressions under salt stress in Chlamydomonas reinhardtii. Int. J. Mol. Sci. 19:2800. doi: 10.3390/ ijms 19092800

Khona, D. K., Shirolikar, S. M., Gawde, K. K., Hom, E., Deodhar, M. A., and D'Souza, J. S. (2016). Characterization of salt stress-induced palmelloids in the green alga, Chlamydomonas reinhardtii. Algal Res 16, 434-448. doi: 10.1016/j. algal.2016.03.035

Klopffleisch, K., Phan, N., Augustin, K., Bayne, R. S., Booker, K. S., Botella, J. R., et al. (2011). Arabidopsis G-protein interactome reveals connections to cell wall carbohydrates and morphogenesis. Mol. Syst. Biol. 7:532. doi: 10.1038/msb. 2011.66

Lalitha, S. (2000). Primer premier 5. Biotech Softw. Internet Rep. 1, 270-272. doi: 10.1089/152791600459894

Lehti-Shiu, M. D., Panchy, N., Wang, P., Uygun, S., and Shiu, S. H. (2017). Diversity, expansion, and evolutionary novelty of plant DNA-binding transcription factor families. Biochim. Biophys. Acta Gene. Regul. Mech. 1860, 3-20. doi: 10.1016/j.bbagrm.2016.08.005

Lehti-Shiu, M. D., Uygun, S., Moghe, G. D., Panchy, N., Fang, L., Hufnagel, D. E., et al. (2015). Molecular evidence for functional divergence and decay of a transcription factor derived from whole-genome duplication in Arabidopsis thaliana. Plant Physiol. 168, 1717-1734. doi: 10.1104/pp.15. 00689
Li, L., Peng, H., Tan, S., Zhou, J., Fang, Z., Hu, Z., et al. (2020). Effects of early cold stress on gene expression in Chlamydomonas reinhardtii. Genomics 112, 1128-1138. doi: 10.1016/j.ygeno.2019.06.027

Love, M. I., Huber, W., and Anders, S. (2014). Moderated estimation of fold change and dispersion for RNA-seq data with DESeq2. Genome Biol. 15:550. doi: 10.1186/s13059-014-0550-8

Luo, M., and Liu, F. (2011). Salinity-induced oxidative stress and regulation of antioxidant defense system in the marine macroalga Ulva prolifera. J. Exp. Mar. Biol. Ecol. 409, 223-228. doi: 10.1016/j.jembe.2011.08.023

Mahong, B., Roytrakul, S., Phaonaklop, N., Wongratana, J., and Yokthongwattana, K. (2012). Proteomic analysis of a model unicellular green alga, Chlamydomonas reinhardtii, during short-term exposure to irradiance stress reveals significant down regulation of several heat-shock proteins. Planta 235, 499-511. doi: 10.1007/s00425-011-1521-x

Meijer, H. J., van Himbergen, J. A., Musgrave, A., and Munnik, T. (2017). Acclimation to salt modifies the activation of several osmotic stress-activated lipid signalling pathways in Chlamydomonas. Phytochemistry 135, 64-72. doi: 10.1016/j.phytochem.2016.12.014

Mendez-Alvarez, S., Leisinger, U., and Eggen, R. I. (1999). Adaptive responses in Chlamydomonas reinhardtii. Int. Microbiol 2, 15-22.

Munns, R., and Tester, M. (2008). Mechanisms salinity tolerance. Annu. Rev. Plant Biol. 59, 651-681. doi: 10.1146/annurev.arplant.59.032607.092911

Neelam, S., and Subramanyam, R. (2013). Alteration of photochemistry and protein degradation of photosystem II from Chlamydomonas reinhardtii under high salt grown cells. Photobiol. B Biol. 124, 63-70. doi: 10.1016/j.jphotobiol. 2013.04.007

Oren, A. (2005). A hundred years of Dunaliella research 1905-2005. Aquat. Biosyst. 1, 1-14. doi: 10.1186/1746-1448-1-2

Perrineau, M. M., Zelzion, E., Gross, J., Price, D. C., Boyd, J., and Bhattacharya, D. (2014). Evolution of salt tolerance in a laboratory reared population of Chlamydomonas reinhardtii. Environ. Microbiol. 16, 1755-1766. doi: 10.1111/ 1462-2920.12372

Pertea, M., Pertea, G. M., Antonescu, C. M., Chang, T. C., Mendell, J. T., and Salzberg, S. L. (2015). StringTie enables improved reconstruction of a transcriptome from RNA-seq reads. Nat. Biotechnol. 33, 290-5. doi: 10.1038/ nbt.3122

Pollock, S. V., Pootakham, W., Shibagaki, N., Moseley, J. L., and Grossman, A. R. (2005). Insights into the acclimation of Chlamydomonas reinhardtii to sulfur deprivation. Photosyn. Res. 86, 475-489. doi: 10.1007/s11120-005-4048-9

Riano-Pachon, D. M., Correa, L. G., Trejos-Espinosa, R., and Mueller-Roeber, B. (2008). Green transcription factors: a Chlamydomonas overview. Genetics 179, 31-39. doi: 10.1534/genetics.107.086090

Ruprecht, C., Proost, S., Hernandez-Coronado, M., Ortiz-Ramirez, C., Lang, D., Rensing, S. A., et al. (2017). Phylogenomic analysis of gene co-expression networks reveals the evolution of functional modules. Plant J. 90, 447-465. doi: 10.1111/tpj. 13502

Salama el, S., Kim, H. C., Abou-Shanab, R. A., Ji, M. K., Oh, Y. K., Kim, S. H., et al. (2013). Biomass, lipid content, and fatty acid composition of freshwater Chlamydomonas mexicana and Scenedesmus obliquus grown under salt stress. Bioprocess Biosyst. Eng. 36, 827-833. doi: 10.1007/s00449-013-0919-1

Shannon, P., Markiel, A., Ozier, O., Baliga, N. S., Wang, J. T., Ramage, D., et al. (2003). Cytoscape: a software environment for integrated models of biomolecular interaction networks. Genome Res. 13, 2498-2504. doi: 10.1101/ gr.1239303

Shetty, P., Gitau, M. M., and Maroti, G. (2019). Salinity stress responses and adaptation mechanisms in eukaryotic green microalgae. Cells 8:1657. doi: 10. 3390/cells8121657

Singh, V., Singh, P. K., Siddiqui, A., Singh, S., Banday, Z. Z., and Nandi, A. K. (2016). Over-expression of Arabidopsis thaliana SFD1/GLY1, the gene encoding plastid localized glycerol-3-phosphate dehydrogenase, increases plastidic lipid content in transgenic rice plants. J. Plant. Res. 129, 285-293. doi: 10.1007/s10265-015-0781-0

Sithtisarn, S., Yokthongwattana, K., Mahong, B., Roytrakul, S., Paemanee, A., Phaonakrop, N., et al. (2017). Comparative proteomic analysis of Chlamydomonas reinhardtii control and a salinity-tolerant strain revealed a differential protein expression pattern. Planta 246, 843-856. doi: 10.1007/ s00425-017-2734-4

Sola, K., Gilchrist, E. J., Ropartz, D., Wang, L., Feussner, I., Mansfield, S. D., et al. (2019). RUBY, a putative galactose oxidase, influences pectin properties and 
promotes cell-to-cell adhesion in the seed coat epidermis of Arabidopsis. Plant Cell 31, 809-831. doi: 10.1105/tpc.18.00954

Thiriet-Rupert, S., Carrier, G., Chenais, B., Trottier, C., Bougaran, G., Cadoret, J. P., et al. (2016). Transcription factors in microalgae: genome-wide prediction and comparative analysis. BMC Genomics 17:282. doi: 10.1186/s12864-0162610-9

Vega, J. M., Garbayo, I., Domínguez, M. J., and Vigara, J. (2006). Effect of abiotic stress on photosynthesis and respiration in Chlamydomonas reinhardtii: induction of oxidative stress. Enzyme. Microb. Technol. 40, 163-167. doi: 10. 1016/j.enzmictec.2005.10.050

Verdu, M., Gomez, J. M., Valiente-Banuet, A., and Schob, C. (2021). Facilitation and plant phenotypic evolution. Trends Plant Sci. 26, 913-923. doi: 10.1016/j. tplants.2021.04.005

Wang, J., Zhang, S., Fu, Y., He, T., and Wang, X. (2020). Analysis of Dynamic Global Transcriptional Atlas Reveals Common Regulatory Networks of Hormones and Photosynthesis Across Nicotiana Varieties in Response to Long-Term Drought. Front. Plant Sci 11:672. doi: 10.3389/fpls.2020. 00672

Wang, N., Qian, Z., Luo, M., Fan, S., Zhang, X., and Zhang, L. (2018). Identification of salt stress responding genes using transcriptome analysis in green alga Chlamydomonas reinhardtii. Int. J. Mol. Sci. 19:3359. doi: 10.3390/ ijms 19113359

Wu, G., Hufnagel, D. E., Denton, A. K., and Shiu, S. H. (2015). Retained duplicate genes in green alga Chlamydomonas reinhardtii tend to be stress responsive and experience frequent response gains. BMC Genomics 16:149. doi: 10.1186/ s12864-015-1335-5

Wu, T. Y., Goh, H., Azodi, C. B., Krishnamoorthi, S., Liu, M. J., and Urano, D. (2021). Evolutionarily conserved hierarchical gene regulatory networks for plant salt stress response. Nat. Plants 7, 787-799. doi: 10.1038/s41477-02100929-7

Xie, Z., Li, D., Wang, L., Sack, F. D., and Grotewold, E. (2010). Role of the stomatal development regulators FLP/MYB88 in abiotic stress responses. Plant J. 64, 731-739. doi: 10.1111/j.1365-313X.2010.04364.x
Yang, W., Wang, F., Liu, L. N., and Sui, N. (2020). Responses of Membranes and the Photosynthetic Apparatus to Salt Stress in Cyanobacteria. Front. Plant Sci. 11:713. doi: 10.3389/fpls.2020.00713

Yang, Z., Li, J. L., Liu, L. N., Xie, Q., and Sui, N. (2019). Photosynthetic Regulation Under Salt Stress and Salt-Tolerance Mechanism of Sweet Sorghum. Front. Plant Sci. 10:1722. doi: 10.3389/fpls.2019.01722

Yokthongwattana, C., Mahong, B., Roytrakul, S., Phaonaklop, N., Narangajavana, J., and Yokthongwattana, K. (2012). Proteomic analysis of salinity-stressed Chlamydomonas reinhardtii revealed differential suppression and induction of a large number of important housekeeping proteins. Planta 235, 649-659. doi: 10.1007/s00425-012-1594-1

Zhang, B., and Horvath, S. (2005). A general framework for weighted gene coexpression network analysis. Stat. Appl. Genet. Mol. Biol. 4:17. doi: 10.2202/ 1544-6115.1128

Zhu, J. K. (2016). Abiotic Stress Signaling and Responses in Plants. Cell 167, 313-324. doi: 10.1016/j.cell.2016.08.029

Conflict of Interest: The authors declare that the research was conducted in the absence of any commercial or financial relationships that could be construed as a potential conflict of interest.

Publisher's Note: All claims expressed in this article are solely those of the authors and do not necessarily represent those of their affiliated organizations, or those of the publisher, the editors and the reviewers. Any product that may be evaluated in this article, or claim that may be made by its manufacturer, is not guaranteed or endorsed by the publisher.

Copyright (c) 2022 Zhang, Xing, Chen, Zhang and Fan. This is an open-access article distributed under the terms of the Creative Commons Attribution License (CC BY). The use, distribution or reproduction in other forums is permitted, provided the original author(s) and the copyright owner(s) are credited and that the original publication in this journal is cited, in accordance with accepted academic practice. No use, distribution or reproduction is permitted which does not comply with these terms. 